\title{
Nonparametric Demand Forecasting with Right Censored Observations
}

\author{
Bin ZHANG ${ }^{1,2}$; Zhongsheng HUA ${ }^{2}$ \\ ${ }^{1}$ Institute for Economics and Lingnan College, Sun Yat-sen University, Guangzhou, China; ${ }^{2}$ School of Management, University of \\ Science and Technology of China, Hefei, China. \\ Email: bzhang3@mail.ustc.edu.cn
}

Received July $17^{\text {th }}, 2009$; revised August $10^{\text {th }}, 2009$; accepted August $18^{\text {th }}, 2009$.

\begin{abstract}
In a newsvendor inventory system, demand observations often get right censored when there are lost sales and no backordering. Demands for newsvendor-type products are often forecasted from censored observations. The Kaplan-Meier product limit estimator is the well-known nonparametric method to deal with censored data, but it is undefined beyond the largest observation if it is censored. To address this shortfall, some completion methods are suggested in the literature. In this paper, we propose two hypotheses to investigate estimation bias of the product limit estimator, and provide three modified completion methods based on the proposed hypotheses. The proposed hypotheses are verified and the proposed completion methods are compared with current nonparametric completion methods by simulation studies. Simulation results show that biases of the proposed completion methods are significantly smaller than that of those in the literature.
\end{abstract}

Keywords: Forecasting, Demand, Censored, Nonparametric, Product Limit Estimator

\section{Introduction}

In a newsvendor inventory system, a decision maker places an order before the selling season with stochastic demand. If too much is ordered, stock is left over at the end of the period, whereas if too little is ordered, sales are lost. The optimal order quantity is often set based on the well-known critical ratio [1], therefore demand observations often get right censored when there are lost sales and no backordering. Because lost sales cannot be observed, the available sales data actually reflect the stock available for sale, rather than the true demand. Demands for newsvendor-type products are often forecasted from censored observations.

The problem of demand forecasting in the presence of stockouts is a well-known problem of handling censored observations, which was recognized by [2]. Approaches of handling censored observations can be divided into two classes: (1) parametric method, which often assumes that the observations come from specific theoretical distribution and then estimate parameters of the assumed distribution by applying maximum likelihood estimation or some updating procedures [3]; This method is often used in density forecasting [4]; (2) nonparametric method, which is often established based on the product limit estimator [5], and attempts to address the problem of the "undefined region" beyond the largest observation when it is censored [6].

Parametric methods for demand forecasting from censored observations have been investigated in [7-14]. These works have been briefly reviewed in [15], and it has been indicated in [15] that it is difficult to determine the shape or family of demand distribution in advance when demand observations are censored.

The product limit (PL) estimator is a nonparametric maximum likelihood estimator of a distribution function based on censored data. If the largest observation is censored, the PL estimator is developed to estimate the left-hand side of demand distribution, but it is undefined for the right-hand side of distribution function. Under the assumption that there are more information besides the censored observations, Lau and Lau [3] and Zhang et al. [15] have investigated the problems of estimating the right-hand side of demand distributions.

Without additional information besides the censored observations, truncation techniques or completion methods are usually employed to define the whole distribution function. Truncation techniques are based on the data-driving rules, which include two common truncation rules: (1) truncating at the largest observation if it is censored, and (2) truncating at $(n-l)$ th order statistics [6]. These truncation rules may intuitively appear to have good properties by avoiding problems in tail, but they will incur large bias because the location of the ignored 
region is a random event. Completion methods aim to redefine the PL estimator beyond the largest observation if it is censored. We will briefly review nonparametric completion methods in the next section.

In this paper, we propose two hypotheses to investigate estimation bias of the PL estimator, and provide three modified completion methods based on the proposed hypotheses. The proposed hypotheses are verified and the proposed completion methods are compared with current nonparametric completion methods in the literature by simulation studies.

The remainder of this paper is structured as follows. We briefly introduce the PL estimator and review current nonparametric completion methods suggested in the literature. Then we propose two hypotheses to investigate estimation bias of the PL estimator, and provide three modified completion methods. We further verify the two hypotheses and compare the proposed completion methods with current nonparametric completion methods by simulation studies. The paper ends with some concluding remarks.

\section{Nonparametric Completion Methods}

In this section, we first introduce the PL estimator in the context of an inventory system, and then we review current nonparametric completion methods for the PL estimator suggested in the literature.

\subsection{Product Limit Estimator}

Let $X_{i}, i=1,2, \ldots, n$, be iid (independent identically-distributed) demand from distribution $F$, and inventory level $Y_{i}, i=1,2, \ldots, n$ be iid from distribution $G$. It is often to assume that both $F$ and $G$ are continuous and defined on the interval $[0, \infty)$. In an inventory system, demand $X_{i}$ is censored on the right by the available inventory level $Y_{i}$, and we observe $Z_{i}=$ $\min \left\{X_{i}, Y_{i}\right\}$ and $\delta_{i}=I\left(X_{i} \leq Y_{i}\right), \quad i=1,2, \ldots, n$, where $I(\cdot)$ stands for the indicator function, and $\delta_{i}$ indicates whether demand observation $Z_{i}$ is censored $\left(\delta_{i}=0\right)$ or $\operatorname{not}\left(\delta_{i}=1\right)$.

Kaplan and Meier [5] introduced the PL estimator for the survival function $S(t)=1-F(t)$, which is estimated as follows:

$$
\hat{S}(t)=\prod_{i=1}^{n}\left(1-\frac{\delta_{i: n}}{n-i+1}\right)^{I\left(z_{i n} \leq t\right)}
$$

where $Z_{i: n}$ denotes the $i$ th ordered observation among all $Z_{i}$, and $\delta_{i: n}$ corresponds to $Z_{i: n}$. From the above definition, it is observed that the PL estimator is undefined beyond the largest observation, i.e., for $t \geq Z_{n: n}$ and $\delta_{n: n}=0$.

\subsection{Review of Current Completion Methods}

To overcome the shortfall of the PL estimator that it is undefined beyond the largest observation, some completion methods are suggested in the literature. Efron [16] introduced the notion of self-consistency, i.e.,

$$
\hat{S}_{E}(t)=0, \text { for } t \geq Z_{n: n} \text {. }
$$

Gill [17] defined the survival function by

$$
\hat{S}_{G}(t)=\left(1-\delta_{n: n}\right) \Pi_{i=1}^{n}\left(1-\frac{\delta_{i: n}}{n-i+1}\right)^{I\left(Z_{i n} \leq t\right)} \text { for } t \geq Z_{n: n}(3)
$$

Chen and Phadia [18] modified it as

$$
\hat{S}_{C}(t)=c\left(1-\delta_{n: n}\right) \Pi_{i=1}^{n-1}\left(1-\frac{\delta_{i: n}}{n-i+1}\right)^{I\left(Z_{i n} \leqslant t\right)} \text { for } t \geq Z_{n: n}
$$

where $c \in[0,1]$ is determined by minimizing the mean squared error loss

$$
E \int_{0}^{\infty}(\hat{F}(t)-F(t))^{2} d F(t)=-\int_{0}^{\infty} E(\hat{S}(t)-S(t))^{2} d S(t)(5)
$$

Clearly, the extreme values of scalar $c$ yield Efron's and Gill's versions, respectively.

Besides the above three constant completion methods, there are two curve completion methods suggested in the literature. Brown et al [19] suggested an exponential completion method as follows:

$$
\hat{S}_{B}(t)=e^{-\lambda_{B} t}, \text { for } t \geq Z_{n: n} .
$$

The parameter $\lambda_{B}$ is set by solving $\hat{S}\left(Z_{n: n}^{-}\right)=e^{-\lambda_{B} Z_{n n}}$, where $Z_{n: n}^{-}=\lim _{\varepsilon>0, \varepsilon \rightarrow 0}\left(Z_{n: n}-\varepsilon\right)$. Let $Z_{(i)}, i=1, \ldots m$, denote the $m$ ordered uncensored demand observations, the remaining $n-m$ observations are censored ones. Moeschberger and Klein [20] attempted to complete $\hat{S}(t)$ by a two-parameter Weibull function as follows:

$$
\hat{S}_{M}(t)=e^{-\lambda_{M} t^{k}}, \text { for } t \geq Z_{n: n}
$$

The two parameters $\lambda_{M}$ and $k$ in Equation (7) are determined by solving $\hat{S}\left(Z_{(m)}\right)=e^{-\lambda_{M} t_{(m)}^{k}}$ and $\hat{S}\left(Z_{(m-1)}\right)=e^{-\lambda_{M} t_{(m-1)}^{k}}$.

When a completion method is used, the bias of $\hat{S}(t)$, $B(t)=E\{\hat{S}(t)\}-S(t)$, is entirely determined by the completion method [21]. For a completion method, it is clear that the "undefined region" has the most contribution to the bias of the PL estimator. One might think that this region could be in some sense ignored, as it is sug- 
gested in truncation techniques. Because the location of this region is a random event, simply ignoring the "undefined region" will result in a large bias [6].

The bias of $\hat{S}_{E}(t)$ is negative and asymptotically zero as $t \rightarrow \infty$, whereas the bias of $\hat{S}_{G}(t)$ is positive and increasing as $t \rightarrow \infty$. The bias using any other completion method will be bounded by the biases of $\hat{S}_{E}(t)$ and $\hat{S}_{G}(t)$ [6]. The bias of $\hat{S}_{C}(t)$ changes from negative to positive and it is increasing as $t \rightarrow \infty$. If an estimator is asymptotically zero as $t \rightarrow \infty$, we say that it has completeness, which is necessary for estimating moments of distribution. $\hat{S}_{E}(t), \hat{S}_{B}(t)$, and $\hat{S}_{M}(t)$ have completeness since they are asymptotically zero as $t \rightarrow \infty$, whereas $\hat{S}_{G}(t)$ and $\hat{S}_{C}(t)$ do not have the completeness. The curve completion methods, $\hat{S}_{B}(t)$, and $\hat{S}_{M}(t)$ satisfy the downward sloping monotonicity of survival function, but the constant completion methods, $\hat{S}_{E}(t), \hat{S}_{G}(t)$ and $\hat{S}_{C}(t)$ do not.

\section{New Completion Methods}

In this section, we first propose two hypotheses to investigate estimation bias of the PL estimator at two special points, and provide three modified completion methods based on the proposed hypotheses. Then we simplify show the nonparametric completion methods by an example.

\subsection{Estimation Bias of the PL Estimator}

If demand observations $X_{i}, i=1,2, \ldots, n$, are observable, then its empirical survival function $\bar{S}(t)$ can be expressed as follows:

$$
\bar{S}(t)=1-\frac{1}{n} \sum_{i=1}^{n} I\left(X_{i} \leq t\right)
$$

Since $X_{n: n}>Z_{n: n}^{-}$, the value of $\bar{S}(t)$ at point $Z_{n: n}^{-}$can be rewritten as

$$
\begin{gathered}
\bar{S}\left(Z_{n: n}^{-}\right)=1-\frac{1}{n} \sum_{i=1}^{n} I\left(X_{i} \leq Z_{n: n}^{-}\right) \\
=\prod_{i=1}^{n-1}\left(1-\frac{1}{n-i+1}\right)^{I\left(X_{i: n} \leq Z_{n: n}^{-}\right)}
\end{gathered}
$$

According to Equation (1), the estimation value of the PL estimator at point $Z_{n: n}^{-}$is

$$
\hat{S}\left(Z_{n: n}^{-}\right)=\Pi_{i=1}^{n-1}\left(1-\frac{\delta_{i: n}}{n-i+1}\right)^{1}
$$

To compare $\bar{S}\left(Z_{n: n}^{-}\right)$and $\hat{S}\left(Z_{n: n}^{-}\right)$, we introduce

$$
\tilde{S}\left(Z_{n: n}^{-}\right)=\prod_{i=1}^{n-1}\left(1-\frac{1}{n-i+1}\right)^{1}
$$

From Equations (9-11), $\hat{S}\left(Z_{n: n}^{-}\right)$can be viewed as a modification of $\bar{S}\left(Z_{n: n}^{-}\right)$by replacing $I\left(X_{i: n} \leq Z_{n: n}^{-}\right)$ by 1 (from Equation (9-11)), and then replacing 1 by $\delta_{i: n}$ (from Equation $(11,10)$ ). By introducing these two replacements, it is clear that $\tilde{S}\left(Z_{n: n}^{-}\right) \leq \bar{S}\left(Z_{n: n}^{-}\right)$and $\tilde{S}\left(Z_{n: n}^{-}\right) \leq \hat{S}\left(Z_{n: n}^{-}\right)$. This indicates that $\tilde{S}\left(Z_{n: n}^{-}\right)$will underestimate $\bar{S}\left(Z_{n: n}^{-}\right), \quad \hat{S}\left(Z_{n: n}^{-}\right)$will overestimate $\tilde{S}\left(Z_{n: n}^{-}\right)$, but $\hat{S}\left(Z_{n: n}^{-}\right)$will underestimate or overestimate $\bar{S}\left(Z_{n: n}^{-}\right)$.

The sign of bias $\hat{S}\left(Z_{n: n}^{-}\right)-\bar{S}\left(Z_{n: n}^{-}\right)$is completely determined by $I\left(X_{i: n} \leq Z_{n: n}^{-}\right)$and $\delta_{i: n}, \quad i=1,2, \ldots, n-1$. Since $I\left(X_{i: n} \leq Z_{n: n}^{-}\right)$and $\delta_{i: n}$ are random variables determined by $X_{i}$ and $Y_{i}, i=1,2, \ldots, n$, the bias is also a random variable and its sign also depends on $X_{i}$ and $Y_{i}, i=1,2, \ldots, n$. In the case when $X_{n-1: n} \leq Z_{n: n}^{-}$ is satisfied and there is at least one censored observation among $Z_{i: n}, i=1,2, \ldots, n-1, \hat{S}\left(Z_{n: n}^{-}\right)$must overestimate $\bar{S}\left(Z_{n: n}^{-}\right)$. We argue that $\delta_{i: n}$ has more important influence on the estimation bias than $I\left(X_{i: n} \leq Z_{n: n}^{-}\right)$ does, i.e., the PL estimator will statistically overestimate at point $Z_{n: n}^{-}$. Based on this perception, we present the following hypothesis:

\section{Hypothesis 1:}

Denote by $B\left(Z_{n: n}^{-}\right)=\hat{S}\left(Z_{n: n}^{-}\right)-\bar{S}\left(Z_{n: n}^{-}\right)$, then $B\left(Z_{n: n}^{-}\right)$ is statistically larger than zero.

Since the PL estimator is a piecewise right continuous function, and the largest uncensored observation $Z_{(m)}$ is a right continuous piecewise point, so the relative estimation bias of the PL estimator at point $Z_{(m)}$ should be statistically smaller than that at point $Z_{n: n}^{-}$. That is, the PL estimator statistically provide more accurate estimation at point $Z_{(m)}$ than at $Z_{n: n}^{-}$. Therefore, we have the following hypothesis:

\section{Hypothesis 2:}

Denote by $R(t)=|\hat{S}(t)-\bar{S}(t)| / \bar{S}(t)$, then $R\left(Z_{(m)}\right)$ is statistically smaller than $R\left(Z_{n: n}^{-}\right)$. 


\subsection{Modified Completion Methods}

In the spirit of the exponential curve completion method suggested by [19], we provide three modified completion methods based on the proposed hypotheses.

Hypothesis 1 implies that the PL estimator will statistically overestimate at point $Z_{n: n}^{-}$. Therefore bias can be reduced if parameter of the exponential curve is set by solving $d \cdot \hat{S}\left(Z_{n: n}^{-}\right)=e^{-\lambda_{D} Z_{n: n}}$ instead of $\hat{S}\left(Z_{n: n}^{-}\right)=e^{-\lambda_{B} Z_{n n}}$, where $d \in(0,1]$ is an adjusted factor for overcoming the overestimation of the PL estimator at point $Z_{n: n}^{-}$. Chen and Phadia [18] proposed an optimal constant completion by setting $\hat{S}\left(Z_{n: n}\right)=c \hat{S}\left(Z_{n: n}^{-}\right)$. Similarly, we set $d=\min (2 c, 1)$. This parameter setting is presented because scalar $d$ should not be larger than one in solving $\lambda_{D}$.

Hypothesis 2 indicates that the relative estimation bias of the PL estimator at point $Z_{(m)}$ is statistically smaller than that at point $Z_{n: n}^{-}$. Therefore bias can be reduced if parameter of the exponential curve is set by solving $\hat{S}\left(Z_{(m)}\right)=e^{-\lambda_{L} Z_{(m)}}$ instead of $\hat{S}\left(Z_{n: n}^{-}\right)=e^{-\lambda_{B} Z_{n n}}$. Since the exponential curve may approximately pass the two points $\left(Z_{(m)}, \hat{S}\left(Z_{(m)}\right)\right)$ and $\left(Z_{n: n}, d \cdot \hat{S}\left(Z_{n: n}^{-}\right)\right)$, the parameter of the exponential curve can also be set as $\lambda_{A}=\left(\lambda_{L}+\lambda_{D}\right) / 2$.

\subsection{An Illustrative Example}

In a case study of a newsvendor inventory system, Lau and Lau [3] presented 20 ordered daily sales observations:
$34,34,37^{*}, 38,44^{*}, 45^{*}, 47^{*}, 50,50,50,60,60^{*}, 65^{*}(8$ times), where asterisk indicates a censored observation, e.g., the third entry ' $37^{*}$ ' means that $Z_{3}=37$ was observed on a day when $Y_{3}=37$, implying that $X_{3} \geq 37$.

For this example, the various aforementioned completion methods are plotted in Figure 1. From Figure 1 it can be observed that, the five curve completion methods satisfy the downward sloping monotonicity of survival function, and that the five curve completion methods and Efron's self-consistent completion have the completeness.

\section{Simulation Studies}

In this section, we verify the two proposed hypotheses and compare the aforementioned completion methods by simulation studies.

\subsection{Simulation Experiments}

In our simulation studies, we design two experiments under an inventory system with some specific distributions as follows:

Experiment 1: Following [22-23], we take demand distribution $F$ to be a Weibull distribution, $F(x)=1-\exp \left(-x^{a}\right)$ for $x \geq 0$ with $a=1$ and 2 . To reflect a variety of censoring distribution patterns, we also follow [23] to take Weibull distribution, $G(y)=$ $1-\exp \left[-(u y)^{b}\right]$ for $x \geq 0$ with $b=a / 2, b=a$, and $b=2 a$, as our censoring inventory distribution. This gives the hazard rate $h(t)=u^{b} t^{b-a}$, which is decreasing for $b / a<1$, constant for $b / a=1$, and increasing $b / a>1$. The scale factor $u$ in $G(y)$ is adjusted in such a way so that the expected stockout probability (ESP)
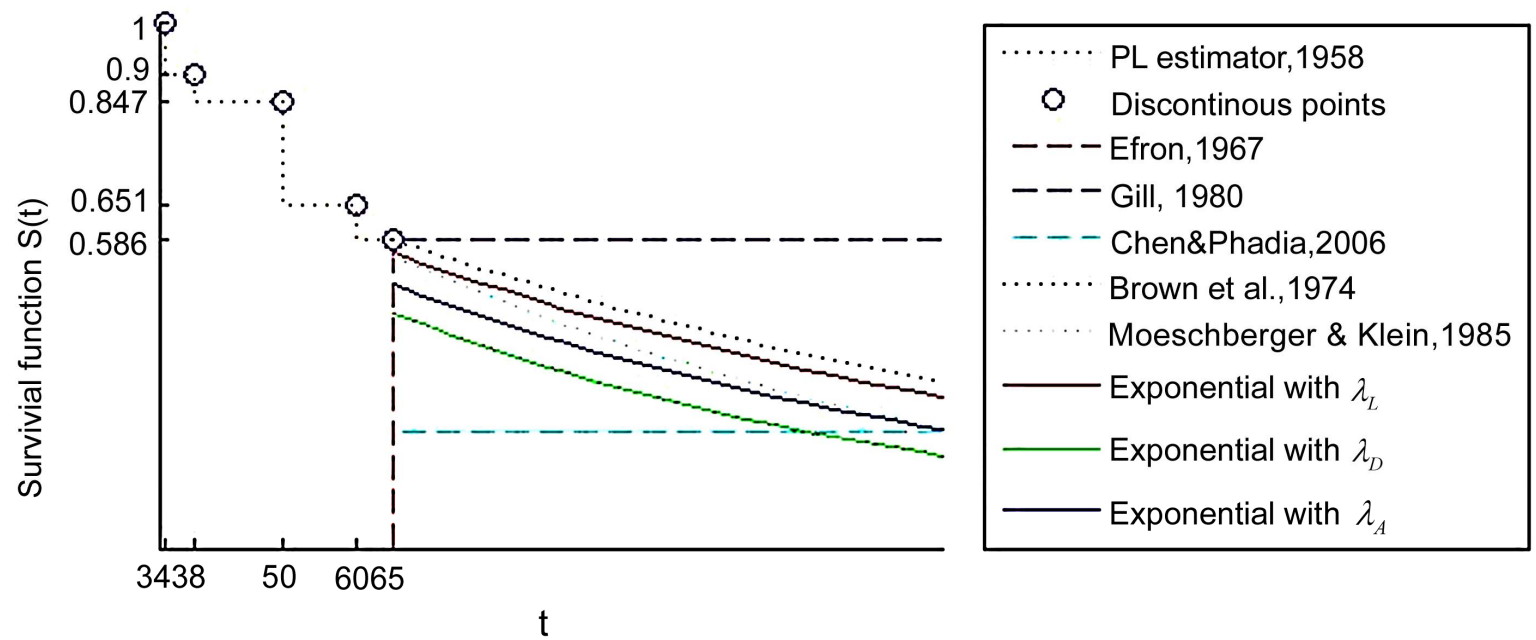

Figure 1. Comparison of the eight completion methods for the PL estimator 
Table 1. Statistical results of $B\left(Z_{n: n}^{-}\right)$in Experiment 1

\begin{tabular}{cccccccc}
\hline & \multicolumn{2}{c}{$b / a=0.5$} & \multicolumn{2}{c}{$b / a=1$} & \multicolumn{2}{c}{$b / a=2$} \\
& $a=1$ & $a=2$ & $a=1$ & $a=2$ & $a=1$ & $a=2$ \\
\hline Mean of $B\left(Z_{n: n}^{-}\right)$ & 0.0082 & 0.0102 & 0.0269 & 0.0259 & 0.0300 & 0.0312 \\
Std. Dev. of $B\left(Z_{n: n}^{-}\right)$ & & 0.0667 & 0.0681 & 0.1075 & 0.1098 & 0.1487 & 0.1511 \\
& Lower & 0.0041 & 0.0060 & 0.0202 & 0.0191 & 0.0208 & 0.0218 \\
95\% C.I. of $B\left(Z_{n: n}^{-}\right)$ & Upper & 0.0123 & 0.0145 & 0.0335 & 0.0327 & 0.0392 & 0.0406 \\
\hline
\end{tabular}

Table 2. Statistical results of $B\left(Z_{n: n}^{-}\right)$in Experiment 2

\begin{tabular}{cccccccc}
\hline & \multicolumn{2}{c}{ ESP =1/3 } & \multicolumn{2}{c}{ ESP =1/2 } & \multicolumn{2}{c}{ ESP =2/3 } \\
& $\sigma=1$ & $\sigma=2$ & $\sigma=1$ & $\sigma=2$ & $\sigma=1$ & $\sigma=2$ \\
\hline Mean of $B\left(Z_{n: n}^{-}\right)$ & 0.0758 & 0.0893 & 0.1637 & 0.1839 & 0.2758 & 0.3144 \\
Std. Dev. of $B\left(Z_{n: n}^{-}\right)$ & 0.0607 & 0.0705 & 0.1053 & 0.1181 & 0.1419 & 0.1464 \\
& Lower & 0.0720 & 0.0849 & 0.1572 & 0.1766 & 0.2670 & 0.3053 \\
95\% C.I. of $B\left(Z_{n: n}^{-}\right)$ & Upper & 0.0796 & 0.0936 & 0.1703 & 0.1912 & 0.2846 & 0.3235 \\
\hline
\end{tabular}

is $1 / 3,1 / 2$, or $2 / 3$. These values thus completely specify the hazard rate. The reader is referred to [23] for further details. This experiment is applied for investigating the case when hazard rate is decreasing, constant or increasing.

Experiment 2: Analogous to [8], we express the relation between demand $X$ and sales $Z$ by writing sales as a random proportion of demand, i.e., $Z_{i}=W_{i} X_{i}$, where $W_{i}$ is a random variable taking values on the interval $[0.5,1]$. For periods with no stockout, $W_{i}=1$, and therefore sales and demand are equal; for periods in which a stockout has occurred, sales will be less than demand with $W_{i}<1$. We assume that stockouts occur in each period (independently) with probability ESP and when a stockout occurs, sales $Z_{i}$ is a random (uniformly distributed) proportion of demand $X_{i}$. In our case studies, we take $F$ to be a lognormal distribution with location parameter 4 , and shape parameter $\sigma=1$ and 2 , and we also set $\mathrm{ESP}=1 / 3,1 / 2$, and $2 / 3$. This experiment is designed for investigating the case when hazard rate changes from increasing to decreasing.

In the above two experiments, we have four different cases in terms of hazard rate: decreasing, constant, increasing, and changing from increasing to decreasing. In comparison with Experiment 1, Experiment 2 makes an additional assumption on the relation between demand and sales, i.e., sales is a random (uniformly distributed) proportion of demand.
Considering the combination of the parameters in the above two experiments, under each of four cases of hazard rate, we have 6 different combinations of the parameters. Under each parameters' combination, we set the number of observations $n=20$, and randomly generate 1000 simulation runs. To ensure the applicability of the completion method suggested by Moeschberger and Klein [20], the number of uncensored observations in each simulation run is restricted to be larger than 3 . For the convenience of comparison, the largest observation in each simulation run is restricted to be a censored one.

\subsection{Hypotheses Verification}

Under each of four cases of hazard rate, we calculate $B\left(Z_{n: n}^{-}\right)$under 1000 simulation runs for verifying Hypothesis 1. Statistical results of $B\left(Z_{n: n}^{-}\right)$are reported in Tables 1 and 2 . In these tables, $95 \%$ C.I. is short for $95 \%$ confidence level.

Results shown in Tables 1 and 2 verify Hypothesis 1 , i.e., the PL estimator statistically overestimates at point $Z_{n: n}^{-}$. Table 1 also illustrates that $B\left(Z_{n: n}^{-}\right)$increases with the increase of $b / a$, this implies that the estimation bias of the case with increasing hazard rate is larger than that of the case with decreasing hazard case. Table 2 also illustrates that $B\left(Z_{n: n}^{-}\right)$increases as the expected stockout probability increases.

To verify the correctness of Hypothesis 2, we calculate $R\left(Z_{n: n}^{-}\right)$and $R\left(Z_{(m)}\right)$ under each of four cases of hazard 
rate. Statistical results of $R\left(Z_{n: n}^{-}\right)$and $R\left(Z_{(m)}\right)$ in the two experiments are reported in Tables 3 and 4, respectively. The last two rows of these two tables present results of paired 2-tailed $t$-tests on $\left(R\left(Z_{n: n}^{-}\right), R\left(Z_{(m)}\right)\right)$. From Tables 3 and 4 , it is observed that the relative estimation bias of the PL estimator of point $Z_{(m)}$ is statistically smaller than that of point $Z_{n: n}^{-}$at the 0.01 significance level. Table 4 also implies that the relative estimation biases of the PL estimator at points $Z_{(m)}$ and $Z_{n: n}^{-}$increase with the increase of the expected stockout probability.

\subsection{Comparison with Current Completion Methods}

In this subsection, we assess performance of the proposed completion methods in terms of integral absolute bias, IAB $=-\int_{0}^{\infty} E|\hat{S}(t)-S(t)| d S(t)$. In our simulation results, Efron and Gill denote Efron's and Gill's completion methods respectively; $\mathrm{CP}$, BHK and MK stand for the completion methods of Chen and Phadia [18], Brown et al [19], and Moeschberger and Klein [20], respectively; Left, Down and Ave represent the proposed exponential curve completion methods with parameter $\lambda_{L}, \lambda_{D}$ and $\lambda_{A}$, respectively.

Results of paired 2-tailed $t$-tests on IAB among the compared eight completion methods under each of four cases of hazard rate are shown in Tables 5-8, respectively. These tables report $t$-statistics on IAB between the row method and column method. One negative $t$-statistic in these tables means that the row method is better than the corresponding column method in terms of IAB, whereas positive $t$-statistic implies that the column method is better than the corresponding row method. $t$-statistic in parentheses represents that the comparison is at the 0.05 significance level; $t$-statistic in square brackets implies that there is no significant difference between the row and column methods; $t$-statistic without parentheses or square brackets expresses that the comparison is at the 0.01 significance level.

According to the following results shown in Tables $5-8$, we come to the following conclusions in terms of IAB: (1) Ave is the leading completion method; (2) Left performs better than the optimal constant completion method CP; (3) $\mathrm{CP}$ is always better than the current curve completion methods (i.e., BHK and MK); (4) Efron and Gill are the two worst completion methods.

Table 3. Statistical results of $R\left(Z_{n: n}^{-}\right)$and $R\left(Z_{(m)}\right)$ in Experiment 1

\begin{tabular}{ccccccc}
\hline & \multicolumn{2}{c}{$b / a=0.5$} & \multicolumn{2}{c}{$b / a=1$} & \multicolumn{2}{c}{$b / a=2$} \\
& $a=1$ & $a=2$ & $a=1$ & $a=2$ & $a=1$ & $a=2$ \\
\hline Mean of $R\left(Z_{n: n}^{-}\right)$ & 0.6077 & 0.6348 & 0.8337 & 0.7906 & 0.7842 & 0.7854 \\
Std. Dev. of $R\left(Z_{n: n}^{-}\right)$ & 0.6497 & 0.6999 & 1.0403 & 1.0034 & 1.2163 & 1.1004 \\
Mean of $R\left(Z_{(m)}\right)$ & 0.4161 & 0.4077 & 0.3987 & 0.3914 & 0.3592 & 0.3609 \\
Std. Dev. of $R\left(Z_{(m)}\right)$ & 0.3011 & 0.3367 & 0.3524 & 0.3743 & 0.3295 & 0.3158 \\
$t$-statistics & 9.4889 & 10.3945 & 13.1338 & 12.6988 & 11.1364 & 12.0961 \\
$P$-value & 0.0000 & 0.0000 & 0.0000 & 0.0000 & 0.0000 & 0.0000 \\
\hline
\end{tabular}

Table 4. Statistical results of $R\left(Z_{n: n}^{-}\right)$and $R\left(Z_{(m)}\right)$ in Experiment 2

\begin{tabular}{ccccccc}
\hline & \multicolumn{2}{c}{ ESP =1/3 } & \multicolumn{2}{c}{ ESP =1/2 } & \multicolumn{2}{c}{ ESP =2/3 } \\
& $\sigma=1$ & $\sigma=2$ & $\sigma=1$ & $\sigma=2$ & $\sigma=1$ & $\sigma=2$ \\
\hline Mean of $R\left(Z_{n: n}^{-}\right)$ & 1.3879 & 1.6892 & 2.8948 & 3.4150 & 4.6486 & 5.7690 \\
Std. Dev. of $R\left(Z_{n: n}^{-}\right)$ & 1.1267 & 1.3526 & 2.0145 & 2.3014 & 2.8261 & 2.9991 \\
Mean of $R\left(Z_{(m)}\right)$ & 0.7473 & 0.9011 & 1.3246 & 1.5836 & 1.7312 & 2.0446 \\
Std. Dev. of $R\left(Z_{(m)}\right)$ & 0.5737 & 0.6427 & 0.9715 & 1.0729 & 1.2893 & 1.3821 \\
$t$-statistics & 17.8034 & 18.6759 & 23.6086 & 23.1638 & 29.4864 & 33.4833 \\
$P$-value & 0.0000 & 0.0000 & 0.0000 & 0.0000 & 0.0000 & 0.0000 \\
\hline
\end{tabular}


Table 5. Results of paried 2-tail $t$-test on IAB in Experiment 1 with $b / a=0.5$

\begin{tabular}{cccccccc}
\hline & Gill & CP & BHK & MK & Left & Down & Ave \\
\hline Efron & 6.1797 & 55.3806 & 30.5818 & 29.3516 & 45.4545 & 42.4871 & 47.1257 \\
Gill & & 28.3342 & 34.3206 & 7.2690 & 27.5968 & 29.7606 & 29.1192 \\
CP & & & $(-2.0951)$ & -35.4652 & 3.6572 & 6.7757 & 9.1816 \\
BHK & & & & -19.0436 & 4.2341 & 8.7696 & 7.6550 \\
MK & & & & & 32.7036 & 30.0723 & 35.1446 \\
Left & & & & & & 2.7375 & 11.0712 \\
Down & & & & & & & $(2.4231)$ \\
\hline
\end{tabular}

Table 6. Results of paried 2-tail $t$-test on IAB in Experiment 1 with $b / a=1$

\begin{tabular}{|c|c|c|c|c|c|c|c|}
\hline & Gill & $\mathrm{CP}$ & BHK & MK & Left & Down & Ave \\
\hline Efron & -13.6287 & 65.0251 & 21.7673 & 31.3227 & 51.5355 & 44.4413 & 52.3274 \\
\hline Gill & & 44.8327 & 51.0237 & 27.3941 & 45.5583 & 47.4534 & 46.4081 \\
\hline $\mathrm{CP}$ & & & -15.5159 & -34.5069 & 6.0864 & (2.5103) & 11.0425 \\
\hline BHK & & & & -5.5002 & 19.4179 & 24.3510 & 22.8946 \\
\hline MK & & & & & 32.5922 & 27.1247 & 34.7498 \\
\hline Left & & & & & & -3.4243 & 9.5686 \\
\hline Down & & & & & & & 10.3352 \\
\hline
\end{tabular}

Table 7. Results of paried 2-tail $t$-test on IAB in Experiment 1 with $b / a=2$

\begin{tabular}{cccccccc}
\hline & Gill & CP & BHK & MK & Left & Down & Ave \\
\hline Efron & -19.4571 & 91.0080 & 32.3403 & 41.2147 & 65.5794 & 65.9205 & 68.7712 \\
Gill & & 57.9369 & 68.1601 & 37.2278 & 60.1003 & 62.0498 & 61.1882 \\
CP & & & -16.7540 & -39.6581 & 7.4145 & 14.3985 & 15.1355 \\
BHK & & & & -7.6101 & 23.9762 & 31.2396 & 28.7295 \\
MK & & & & & 36.1403 & 39.3110 & 41.0343 \\
Left & & & & & & 7.4387 & 17.7037 \\
Down & & & & & & & {$[1.6798]$} \\
\hline
\end{tabular}

Table 8. Results of paried 2-tail $\boldsymbol{t}$-test on IAB in Experiment 2

\begin{tabular}{|c|c|c|c|c|c|c|c|}
\hline & Gill & $\mathrm{CP}$ & BHK & MK & Left & Down & Ave \\
\hline Efron & -43.8358 & $(2.3348)$ & -18.9354 & $(-2.3319)$ & 19.2388 & -3.3448 & 16.5648 \\
\hline Gill & & 48.8904 & 45.7360 & 44.1637 & 46.6532 & 45.9931 & 47.0639 \\
\hline $\mathrm{CP}$ & & & -33.3675 & -4.8638 & 18.7680 & -12.9462 & 19.9046 \\
\hline BHK & & & & 19.8107 & 31.3266 & 35.5785 & 34.2751 \\
\hline MK & & & & & 21.2063 & {$[-1.7481]$} & 19.4924 \\
\hline Left & & & & & & -21.9985 & $(-2.4131)$ \\
\hline Down & & & & & & & 26.5872 \\
\hline
\end{tabular}

\section{Conclusions}

Demands for newsvendor-type products are often forecasted from censored observations. The Kaplan-Meier product limit estimator is the well-known nonparametric method to deal with censored data, but it is undefined beyond the largest observation. In this paper, we propose two hypotheses to investigate estimation bias of the PL estimator, and provide three modified completion methods based on the proposed hypotheses.

Simulation results show that biases of the proposed completion methods are significantly smaller than that of the completion methods in the literature. According to these results, we know that the proposed completion methods can improve demand forecasting with right censored observations. We also show that simulation is a useful way to verify probability result which is difficult to be proved by using classical statistical theory and methods.

The developed methods are easy to implement in software packages. Many forecasting techniques have been integrated into enterprise software packages such as management information systems, enterprise resources planning systems, decision support systems. The proposed forecasting techniques in this paper are simple and easily implemented in enterprise software packages. 


\section{Acknowledgements}

This work is supported by national Natural Science Foundation of China (No. 70801065).

\section{REFERENCES}

[1] B. Zhang, X. Xu, and Z. Hua, "A binary solution method for the multi-product newsboy problem with budget constraint," International Journal of Production Economics, Vol. 117, No. 1, pp. 136-141, January 2009.

[2] G. Hadley and T. M. Whitin, "Analysis of inventory systems," Prentice-Hall, Englewood Cliffs, 1963.

[3] H. S. Lau and A. H. L. Lau, "Estimating the demand distributions of single-period items having frequent stockouts," European Journal of Operational Research, Vol. 92, No. 2, pp. 254-265, July 1996.

[4] Z. Hua and B. Zhang, "Improving density forecast by modeling asymmetric features: An application to S\&P500 returns," European Journal of Operational Research, Vol. 185, No. 2, pp. 716-725, March 2008.

[5] E. L. Kaplan and P. Meier, "Nonparametric estimation from incomplete observations," Journal of the American Statistical Association, Vol. 53, No. 282, pp. 457-481, June 1958.

[6] B. Gillespie, J. Gillespie, and B. Iglewicz, "A comparison of the bias in four versions of the product-limit estimator," Biometrika, Vol. 79, No. 1, pp. 149-155, March 1992.

[7] S. A. Conrad, "Sales data and the estimation of demand," Operational Research Quarterly, Vol. 27, No. 1, pp. 123-127, 1976.

[8] W. Wecker, "Predicting demand from sales data in the presence of stockouts," Management Science, Vol. 24, No. 10, pp. 1043-1054, June 1978.

[9] D. B. Braden and M. Freimer, "Informational dynamics of censored observations," Management Science, Vol. 37, No. 11, pp. 1390-1404, November 1991.

[10] N. S. Agrawal and A. Smith, "Estimating negative binomial demand for retail inventory management with unobservable lost sales," Naval Research Logistics, Vol. 43, No. 6, pp. 839-861, September 1996.

[11] P. C. Bell, "Adaptive sales forecasting with many stockouts," Journal of the Operational Research Society, Vol. 32, No. 10, pp. 865-873, October 1981.
[12] P. C. Bell, "A new procedure for the distribution of periodicals," Journal of the Operational Research Society, Vol. 29, No. 5, pp. 427-434, May 1978.

[13] P. C. Bell, "Forecasting demand variation when there are stockouts," Journal of the Operational Research Society, Vol. 51, No. 3, pp. 358-363, March 2000.

[14] X. Ding, "Estimation and optimization in discrete inventory models," Ph.D. thesis, The University of British Columbia, Vancouver, Canada, 2002.

[15] W. Zhang, B. Zhang, and Z. Hua, "Quasi-bootstrap procedure for forecasting demand from sales data with stockouts," in Proceedings of the 38th International Conference on Computers and Industrial Engineering, Vol. 1-3, pp. 423-429, October 2008.

[16] B. Efron, "The two sample problem with censored data," in Proceedings of the 5th Berkeley Symposium on Mathematical Statistics and Probability, Vol. 4, pp. 831-852, 1967.

[17] R. D. Gill, "Censoring and stochastic integrals," Mathematical Centre Tract No. 124, Mathematisch Centrum, Amsterdam, 1980.

[18] Z. Chen and E. Phadia, "An optimal completion of the product limit estimator," Statistics \& Probability Letters, Vol. 76, No. 9, pp. 913-919, May 2006.

[19] B. W. Brown, M. Jr. Hollander, and R. M. Korwar, "Nonparametric tests of independence for censored data, with applications to heart transplant studies," in: F. Proschan and R. J. Serfling, (Eds.), "Reliability and biometry: statistical analysis of lifelength," Philadelphia: Society for Industrial and Applied Mathematics, pp. 291-302, 1974.

[20] M. L. Moeschberger and J. P. Klein, "A comparison of several methods of estimating the survival function when there is extreme right censoring," Biometrics, Vol. 41, No. 1, pp. 253-259, March 1985.

[21] P. Meier, "Estimation of a distribution function from incomplete observations," in: J. Gani (Eds.), "Perspectives in probability and statistics," Applied Probability Turst, Sheffield, England, pp. 67-87, 1975.

[22] J. H. J. Geurts, "Some small-samplel nonproportional hazards results for the Kaplan-Meier estimator," Statistica Neerlandica, Vol. 39, No. 1, pp. 1-13, March 1985.

[23] J. H. J. Geurts, "On the small-sample performance of Efron's and of Gill's version of the product limit estimator under nonproportional hazards," Biometrics, Vol. 43, No. 3, pp. 683-692, September 1987. 